\title{
EFECTO DE LA TEMPERATURA SOBRE EL DESARROLLO DE Eriopis connexa connexa (Germar) (COLEOPTERA: COCCINELLIDAE)
}

\section{TEMPERATURE EFFECT ON THE DEVELOPMENT OF Eriopis connexa connexa (Germar) (COLEOPTERA: COCCINELLIDAE)}

\author{
Héctor William Duarte', Ingeborg Zenner de Polanía²
}

${ }^{1}$ I.A. M.Sc., Docente, Universidad de Ciencias Aplicadas y Ambientales, U.D.C.A, Facultad de Ingeniería Agronómica, wduarte@ udca.edu.co, Calle 222 No. 55-37 Bogotá D.C., ${ }^{2}$ I.A., Ph.D., Docente U.D.C.A, Facultad de Ingeniería Agronómica, izenner@ udca.edu.co

Rev. U.D.C.A Act. \& Div. Cient. 12 (2): 135-145, 2009

\section{RESUMEN}

El manejo apropiado de poblaciones de controladores biológicos en campo y en laboratorio requiere conocimiento de los aspectos biológicos de las mismas. El propósito de este trabajo fue el de evaluar el ajuste del desarrollo dependiente de la temperatura de Eriopis connexa a los modelos matemáticos existentes, para determinar las temperaturas máxima, mínima y óptima para la evolución de cada uno de sus estados. Partiendo de una cría de laboratorio, se estimó la duración de cada uno de los estados inmaduros e instares del depredador en las temperaturas $14 ; 17,6 ; 25,6 ; 30 ; 32$ y $34^{\circ} \mathrm{C}$. Se encontró que la tasa de desarrollo de los estados inmaduros de $E$. connexa, se ajustó muy bien a los modelos de Logan y Brière, obteniéndose para huevo valores de 11,$3 ; 34$ y $33^{\circ} \mathrm{C}$, en umbrales mínimo, máximo y temperatura óptima, respectivamente. Los valores correspondientes para los otros estados fueron los siguientes: la mínima para larva osciló entre 7,7 a $11,9^{\circ} \mathrm{C}$; la máxima fue de $34^{\circ} \mathrm{C}$ y la óptima varió entre 31,9 a $32,9^{\circ} \mathrm{C}$; la pupa presentó un mínimo de $14,5^{\circ} \mathrm{C}$, un máximo de $34^{\circ} \mathrm{C}$ y un óptimo de $34^{\circ} \mathrm{C}$. Para la cría y el establecimiento de este depredador, los estados inmaduros crecieron de forma óptima a temperaturas constantes muy cercanas a los $30^{\circ} \mathrm{C}$. Su establecimiento, se puede limitar a temperaturas inferiores a $14,5^{\circ} \mathrm{C}$ o cuando éstas se acercan a $34^{\circ} \mathrm{C}$.
Palabras clave: Ciclo de vida, depredadores, Coccinéllidos, modelos de desarrollo.

\section{SUMMARY}

The appropriate management of populations of biocontrol agents in field and laboratory, requires the knowledge of their biological aspects. The purpose of this research was to evaluate the adjustment of development, dependent on temperature of the predator Eriopis connexa, to the existing mathematical models, in order to determine maximum, minimum and optimal temperatures for the development of each of its stages. Starting with a laboratory colony, the duration of each immature stage and instar at temperatures of 14; 17.6; $25.6 ; 30 ; 32 \mathrm{y} 34^{\circ} \mathrm{C}$ was determined. It was found that the developmental rate of the immature stages of $E$. connexa fits very well the Logan and Brière model, obtaining respectively for the egg stage values of $11.3 ; 34$ and $33^{\circ} \mathrm{C}$ for the minimum, maximum and optimal temperature threshold. The corresponding values for the other stages were: for the larva the minimum oscillated between 7.7 and $11.9^{\circ} \mathrm{C}$, the maximum was $34^{\circ} \mathrm{C}$ and the optimal varied between 31.9 and $32.9^{\circ} \mathrm{C}$. The pupa presented a minimum of $14.5^{\circ} \mathrm{C}$, a maximum of $34^{\circ} \mathrm{C}$ and an optimum of $34^{\circ} \mathrm{C}$. For rearing and establishment of the predator, the immature stages develop in an optimal manner at constant temperatures very near to $30^{\circ} \mathrm{C}$, but 
its establishment can be limited at temperatures lower than $14.5^{\circ} \mathrm{C}$ or near $34^{\circ} \mathrm{C}$.

Key words: Life cycle, predator, coccinelids, developmental models.

\section{INTRODUCCIÓN}

En la Sabana de Bogotá y en el altiplano Cundiboyacense, se observa de manera frecuente el coccinélido, Eriopis connexa connexa (Germar) (Coleoptera), que se alimenta de insectos plaga, figurando los lepidópteros de la familia Gelechiidae, la polilla guatemalteca de la papa, Tecia solanivora (Povolny) (López-Ávila E EspitiaMalagón, 2000) y la palomilla de la papa, Phtorimaea operculella (Zeller) y la chinche de los pastos, Collaria scenica Stal. (Hemiptera: Miridae); algunos áfidos (Homoptera: Aphididae), Rhopalosiphum maidis (Fitch), Myzus persicae Sulzer, Brevicoryne brassicae (L.) y el ácaro Tetranychus urticae Koch (Acari: Tetranychidae), (Vergara, 1993). En cultivos en zonas templadas, se ha reportado $E$. connexa como enemigo natural de Myzus nicotianae Blackman y M. persicae (Ohashi $\mathcal{E}$ Urdampilleta, 2003) y de huevos de Alabama argillacea (Hübner) (Lepidoptera: Noctuidae) (Arias de Lavalle E Costamagna 1994). En cultivos tropicales, como soya, girasol o alfalfa, se tienen reporte de su acción controladora sobre poblaciones de otros lepidópteros, Rachiplusia nu (Guenée) y Pseudoplusia includens (Walker) (Noctuidae) (Rocha de Moraes et al. 1991).

El conocimiento de la capacidad de adaptación de los enemigos naturales de las plagas a las condiciones climáticas, determina la posibilidad de su empleo en lugares en donde se desarrollan las especies que se desean controlar. El control biológico ya sea por introducción, aumento o conservación, se facilita cuando las respuestas climáticas de los agentes de control son conocidas (Roy et al. 2002).

Estudios con depredadores y sus presas han mostrado que su desarrollo se ve afectado con el cambio de temperatura, de humedad, de fotoperíodo y del huésped o de presa (Obrycki E Kring, 1998). Las características termales varían entre especies, poblaciones, estados y la fuente de alimento (Roy et al. 2002; Zilahi-Balogh et al. 2003; Atlihan $\mathcal{E}$ Chi, 2008). Estos estudios son básicos para comprender la dinámica depredador-presa, la biología y la distribución de las especies; mejorar la eficiencia de las crías masivas; predecir el progreso y la actividad en el campo y útiles para los modelos que estiman el crecimiento, el desarrollo y la reproducción de los insectos (Zilahi-Balogh et al. 2003).

Se han construido modelos matemáticos que describen el efecto de la temperatura sobre el desarrollo. Inicialmente, se propuso un modelo lineal, útil para la tasa de desarrollo frente al incremento de la temperatura, para cada uno de los estados de los insectos (Morris \& Fulton 1970). Sin embargo, este modelo solo describe una fase lineal y proporciona un dato teórico de umbral mínimo de temperatura que, obtenido por interpolación, no se ajusta a los valores observados; además, no provee la temperatura óptima, ni los umbrales máximo y mínimo. Logan et al. (1976) plantearon un modelo analítico no lineal para describir la tasa de desarrollo dependiente de la temperatura, útil para rangos de temperatura óptima o para las que se encuentran fuera de ellos.

Ante la dificultad del modelo de Logan para medir el umbral inferior de temperatura, Lactin et al. (1995) propusieron dos modificaciones al modelo de Logan. Otros dos patrones matemáticos fueron sugeridos posteriormente por Brière et al. (1999), que tienen parámetros ecológicos que se pueden medir y que, además, pueden ser estimados por regresión no lineal. Investigaciones posteriores con el coccinélido Stethorus punctillum (Weise), depredador de Tetranychus mcdanieli McGregor, evaluaron el ajuste de las tasas de crecimiento de ambas especies a once modelos matemáticos de desarrollo dependiente de la temperatura, hallando, que, la segunda ecuación de Lactin et al. (1995) y la primera ecuación de Brière son las que mejor describen el comportamiento de las dos especie (Roy et al. 2002).

Otro ejemplo del ajuste al modelo de Logan es el aplicado en programas de control biológico por Herrera et al. (2005) con el crisómelido (Coleoptera) Diorhabda elongata (Brullé), utilizado para el control de Tamarix sp., una arvense de áreas riparias.

En cuanto a $E$. connexa, las observaciones de tasas de desarrollo, dependiente de la temperatura, se ajustan al modelo lineal de grados-día, reflejando que requiere 259 grados-día para completar su desarrollo y un umbral inferior de desarrollo a $9,2^{\circ} \mathrm{C}$. A partir de estos resultados, los autores interpretaron que ésta es 
la especie consumidora de áfidos que mejor se adapta a condiciones de temperaturas más bajas (Miller $\mathcal{E}$ Paustian, 1992).

El propósito del presente estudio fue determinar el efecto de la temperatura en el desarrollo del depredador $E$. connexa connexa y evaluar el ajuste de los resultados a los modelos matemáticos que puedan describir, apropiadamente, este comportamiento.

\section{MATERIALES Y MÉTODOS}

Este estudio, se desarrolló en el laboratorio de entomología de la Universidad de Ciencias Aplicadas y Ambientales U.D.C.A, ubicada en Bogotá D.C., a $4^{\circ} 48^{\prime}$ latitud norte y $74^{\circ} 03^{\prime}$ longitud oeste, con una altitud de $2.570 \mathrm{msnm}$. Los adultos de $E$. connexa fueron colectados en el invernadero de la U.D.C.A y llevados al laboratorio, como pie de cría; se aparearon, obteniendo una primera generación, cuya descendencia se utilizó para iniciar los diferentes ensayos. Se alimentaron con áfidos de las especies Myzus persicae (Sulzer) y Toxoptera aurantii (Boyer de Fonscolombe), criados sobre plantas de rosa variedad Manneti y sobre una especie del género Pyracantha (Rosaceae), respectivamente.

Efecto de la temperatura: En una incubadora, se determinó el ciclo de vida de $E$. connexa a diferentes temperaturas: $14 \pm 2^{\circ} \mathrm{C} ; 17,6 \pm 2,5^{\circ} \mathrm{C} ; 25,6 \pm 2^{\circ} \mathrm{C}$; $30 \pm 2^{\circ} \mathrm{C} ; 32 \pm 1,7^{\circ} \mathrm{C}$ y $34 \pm 1,5^{\circ} \mathrm{C}$. Un número variable de huevos de edad, inferior a 24 horas de puestos, se colocó dentro de la incubadora, en varios momentos, siendo considerado cada uno de éstos como una repetición. Para cada temperatura, se observó y se registró la eclosión y todas las mudas que se presentaban, diariamente. Cada larva, se ubicó en un recipiente de plástico transparente, con tapa perforada y de capacidad de $50 \mathrm{~mL}$. Todos los días se suministraron ninfas y adultos de $M$. persicae como alimento.

Con los datos de tiempo de desarrollo y de mortalidad, se calculó $d$ para cada estado, de acuerdo con el método propuesto por Logan et al. (1976):

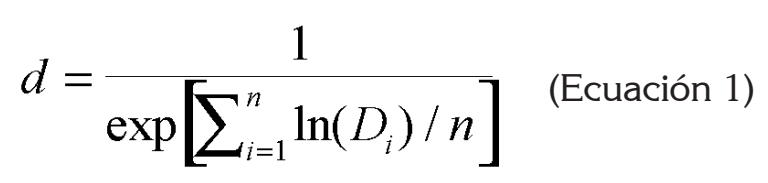

En donde $\boldsymbol{d}$ es la tasa de desarrollo promedio, $\boldsymbol{D}_{i}$, el tiempo de desarrollo observado y $\boldsymbol{n}$, es el tamaño de la muestra. Posteriormente, se construyeron las curvas de tasa de desarrollo y la curva de supervivencia. Con la primera curva, se estimaron los parámetros necesarios para el ajuste a los diferentes modelos de curvas de tasas de desarrollo dependientes de la temperatura, aplicando el método de regresión no lineal iterativa, basado sobre el algoritmo de Marquardt (SAS Institute, 2000). Se consideraron dos modelos por ser los que mejor ajuste presentaron a los datos obtenidos en los ensayos de laboratorio. El primero de ellos fue el de Logan et al. (1976), descrito por la siguiente ecuación:

$$
d(T)=\Psi\left[\exp (\rho T)-\exp \left(\rho T_{M}-\tau\right)\right] \quad \text { (Ecuación 2) }
$$

En donde $d(T)$ es el desarrollo dependiente de la temperatura; $\Psi$, la tasa de desarrollo a la temperatura base; $\rho$, la tasa de incremento hasta la temperatura óptima; $T_{M}$, la máxima temperatura, a la cual, el proceso de vida no puede continuar por un periodo prolongado de tiempo y $\tau$ es una variable resultante del cociente entre la diferencia entre $T_{M}$ y $T$ y la diferencia entre la $T_{M}$ y la temperatura óptima. El segundo fue el modelo descrito por la ecuación de Brière et al. (1999):

$$
R(T)=\operatorname{aT}\left(T-T_{0}\right)\left(T_{L}-T\right)^{1 / 2} \quad(\text { Ecuación 3) }
$$

En donde $R$ es la tasa de desarrollo y es una función positiva de la temperatura $T$ en grados centígrados; $T_{L \text {, }}$ la temperatura letal sobre el umbral; $T_{o}$ la temperatura umbral inferior de desarrollo y a es una constante empírica. Adicionalmente, se realizaron validaciones sobre los residuales de cada estado en cada uno de los dos modelos evaluados para verificar el ajuste.

\section{RESULTADOS Y DISCUSIÓN}

Efecto de la temperatura sobre la supervivencia de cada estado y sobre el desarrollo: La duración en días de los estados de $E$. connexa para cada una de las temperaturas, se presenta resumido en la tabla 1; además, se incluye el número total de individuos evaluados en cada estado y la proporción total del ciclo de vida, que tomó cada uno de ellos. Se observa que el desarrollo no se completó a las temperaturas de 14 y $34^{\circ} \mathrm{C}$ y que el tiempo se redujo al incrementarse la temperatura entre 17,6 y $30^{\circ} \mathrm{C}$, pero se incrementa nuevamente a los $32^{\circ} \mathrm{C}$, 
Tabla 1. Duración promedia, en días, de cada uno de los estados de desarrollo de E. connexa a diferentes temperaturas.

\begin{tabular}{|c|c|c|c|c|c|c|}
\hline \multirow{2}{*}{ Estado o Instar } & \multicolumn{6}{|c|}{ Temperatura $\left({ }^{\circ} \mathrm{C}\right)$} \\
\hline & $14 \pm 2$ & $17,6 \pm 2,5$ & $25,6 \pm 2$ & $30 \pm 2$ & $32 \pm 1,7$ & $34 \pm 1,5$ \\
\hline Huevo & $\begin{array}{c}15,3 \pm 5,45 \\
(760)\end{array}$ & $\begin{array}{c}8,5 \pm 0,5 \\
(103) \\
16,5 \%\end{array}$ & $\begin{array}{c}4,1 \pm 0,3 \\
(194) \\
18,9 \%\end{array}$ & $\begin{array}{c}2,8 \pm 0,4 \\
(82) \\
17,6 \%\end{array}$ & $\begin{array}{c}2,5 \pm 1,7 \\
(4) \\
14,4 \%\end{array}$ & * \\
\hline 1er. instar & $\begin{array}{c}7,8 \pm 0,4 \\
(395)\end{array}$ & $\begin{array}{c}11,3 \pm 2,1 \\
(82) \\
21,9 \%\end{array}$ & $\begin{array}{c}2,9 \pm 0,7 \\
(163) \\
13,2 \%\end{array}$ & $\begin{array}{c}2,5 \pm 0,7 \\
(71) \\
15,8 \%\end{array}$ & $\begin{array}{c}2,4 \pm 0,8 \\
(7) \\
13,7 \%\end{array}$ & * \\
\hline 20. instar & $\begin{array}{c}10,2 \pm 3,6 \\
(90)\end{array}$ & $\begin{array}{c}8,0 \pm 2,7 \\
(74) \mathrm{De} \\
15,5 \%\end{array}$ & $\begin{array}{c}2,4 \pm 1,0 \\
(140) \\
10,9 \%\end{array}$ & $\begin{array}{c}2,0 \pm 0,7 \\
(64) \\
12,6 \%\end{array}$ & $\begin{array}{c}1,9 \pm 0,9 \\
(7) \\
10,7 \%\end{array}$ & * \\
\hline 3er. instar & $\begin{array}{c}15,3 \pm 5,5 \\
(27)\end{array}$ & $\begin{array}{c}7,3 \pm 2,1 \\
(43) \\
14,1 \%\end{array}$ & $\begin{array}{c}3,3 \pm 1,3 \\
(127) \\
15,1 \%\end{array}$ & $\begin{array}{c}2,2 \pm 1,0 \\
(49) \\
13,7 \%\end{array}$ & $\begin{array}{c}2,7 \pm 0,7 \\
\quad(9) \\
15,4 \%\end{array}$ & * \\
\hline 40. instar & $\begin{array}{c}16,2 \pm 1,6 \\
(5)\end{array}$ & $\begin{array}{c}8,5 \pm 3,9 \\
(19) \\
16,6 \%\end{array}$ & $\begin{array}{c}5,0 \pm 1,1 \\
(112) \\
229 \%\end{array}$ & $\begin{array}{c}3,6 \pm 0,9 \\
(44) \\
22,6 \%\end{array}$ & $\begin{array}{c}5,0 \pm 0 \\
(5) \\
29,2 \%\end{array}$ & * \\
\hline Pupa & * & $\begin{array}{c}7,9 \pm 1,6 \\
(27) \\
15,4 \%\end{array}$ & $\begin{array}{c}4,1 \pm 0,8 \\
(102) \\
19,0 \%\end{array}$ & $\begin{array}{c}2,8 \pm 0,8 \\
(50) \\
17,7 \%\end{array}$ & $\begin{array}{c}2,9 \pm 0,4 \\
(8) \\
16,6 \%\end{array}$ & * \\
\hline Preimaginal & * & $\begin{array}{c}50,4 \pm 3,5 \\
(36) \\
100 \%\end{array}$ & $\begin{array}{c}21,6 \pm 1,5 \\
(106) \\
100 \%\end{array}$ & $\begin{array}{c}15,9 \pm 1,2 \\
(56) \\
100 \%\end{array}$ & $\begin{array}{c}18,3 \pm 5,3 \\
\quad(8) \\
100 \%\end{array}$ & * \\
\hline $\begin{array}{l}\text { Macho } \\
\text { Adulto }\end{array}$ & & ** & $\begin{array}{c}44,8 \pm 16,5 \\
(21)\end{array}$ & $\begin{array}{c}22,2 \pm 6,5 \\
(11)\end{array}$ & ** & * \\
\hline $\begin{array}{l}\text { Hembra } \\
\text { Adulta }\end{array}$ & & ** & $\begin{array}{c}46,8 \pm 11,9 \\
(28)\end{array}$ & $\begin{array}{c}26,8 \pm 9,9 \\
(17)\end{array}$ & ** & * \\
\hline
\end{tabular}

* No se completó el desarrollo del estado.

${ }^{* *}$ No se registraron datos.

Los valores entre paréntesis corresponden al número de individuos observados en cada estado. Los porcentajes hacen referencia a la proporción de cada estado dentro de la fase preimaginal.

para el caso particular del tercer y cuarto instar larval, de la pupa y del total preimaginal.

El estado adulto solamente se alcanzó con temperaturas superiores a los $14^{\circ} \mathrm{C}$ y su duración se registró, únicamente, para las temperaturas de 25,6 y $30^{\circ} \mathrm{C}$. Se debe tener en cuenta que se descartaron datos extremos con fines estadísticos, por ser de muy baja frecuencia.
En cuanto a la duración de cada uno de los dos sexos, se registró que tanto para $25,6^{\circ} \mathrm{C}$ como para $30^{\circ} \mathrm{C}$ la duración de las hembras no es significativamente diferente a la de los machos, al 95\% de confianza de una prueba de $\mathrm{T}$ de Student $(\mathrm{T}=1,04 ; \mathrm{P}=0,151 ; \mathrm{gl}=22$ para $25,5^{\circ} \mathrm{C}$ y $\mathrm{T}=0,96 ; \mathrm{P}=0,170$ y $\mathrm{gl}=20$ para $30^{\circ} \mathrm{C}$ ). 
Estos resultados contrastan con los obtenidos por Miller (1995), quien señala que los huevos de E. connexa pueden ser almacenados a temperaturas de $4^{\circ} \mathrm{C}$, hasta por diez días y las pupas a la misma temperatura hasta por cuatro semanas. También, sorprende el hecho de que $E$. connexa se halla con mucha frecuencia en agroecosistemas de clima frío, como lo es el altiplano Cundiboyasense, principalmente, en las épocas secas. Esto, quizá, tenga explicación, porque en las condiciones experimentales la temperatura fue relativamente constante, mientras que en el campo, durante los períodos de verano se tienen fuertes oscilaciones de temperatura entre el día y la noche, razón por las cuales las bajas temperaturas no se tienen por periodos superiores a las quince horas. Desde el punto de vista ecológico esta adaptabilidad le permite a E. connexa prevalecer frente a cambios, relativamente drásticos, en las condiciones de temperatura, dentro del rango mencionado. La supervivencia del tercer instar a la temperatura que normalmente no se encuentra en el campo, pero sí bajo techo, puede ser también una estrategia que le permite a esta especie prevalecer cuando la temperatura se incrementa, al tener una fase de su ciclo de desarrollo tolerante a los momentos en que las condiciones ambientales salen de su rango óptimo. Otra explicación podría ser el hecho del uso de individuos provenientes de invernadero, quizás adaptadas a condiciones de más altas temperaturas que las poblaciones de este depredador de campo.

De acuerdo con lo datos presentados en la tabla 2, todos los estados e instares larvales mostraron baja supervivencia a $14^{\circ} \mathrm{C}$, exceptuando el huevo. Para todos ellos, la supervivencia fue superior al $80 \%$ a temperaturas entre 17,6 y $30^{\circ} \mathrm{C}$, siendo esa última la que menor mortalidad ocasionó, en particular, al estado de pupa. También, se observó que a $32^{\circ} \mathrm{C}$ solo el tercer instar muestra una sobrevivencia óptima (92\%). En cuanto a las condiciones de cría artificial, se tiene que, tomando los resultados de supervivencia, la temperatura puede oscilar entre los 17,6 y $30^{\circ} \mathrm{C}$, para los estados de larva y pupa, mientras que para el huevo debe estar entre los 25,6 y $30^{\circ} \mathrm{C}$, siendo la última la que mayor supervivencia pudo garantizar.

Los valores de supervivencia de huevo a adulto son bajos comparados con los obtenidos por Miller $E$ Paustian (1992), en los que la supervivencia superó el $67 \%$, a partir de los $14^{\circ} \mathrm{C}$, alcanzando un $95 \%$ a los $18^{\circ} \mathrm{C}$ y, desde esta temperatura, la supervivencia desciende, levemente, hasta el $86 \%$, en la temperatura de $34^{\circ} \mathrm{C}$, sin mostrar nunca niveles tan bajos, como los obtenidos en el presente trabajo. Lo anterior se puede explicar por la diferencia entre las poblaciones del coccinélido evaluado y también a las condiciones experimentales, ya que el ensayo reportado se realizó con depredadores provenientes del centro de Argentina, en donde las oscilaciones en la temperatura no son tan abruptas durante el final de la primavera y el verano, épocas en que abunda este depredador. El experimento de Miller E Paustian (1992) fue realizado a un fotoperíodo de 16:8 (Luz:Oscuridad), siendo posible además, que la dieta suministrada tenga influencia en la supervivencia, al ser la utilizada, en esta investigación, presas que no proporcionen todos los requerimientos nutricionales del depredador, como sí pudieron serlo los áfidos del trigo, Diuraphis noxia (Mordvilko) y Rophalosiphum padi (L.), empleados por los autores citados. Los rangos de tiempo

Tabla 2. Supervivencia de los estados e instares larvales de E. connexa para cada una de las temperaturas evaluadas.

\begin{tabular}{|c|c|c|c|c|c|c|c|c|}
\hline \multirow{2}{*}{ Temperatura } & \multicolumn{7}{|c|}{ Supervivencia } \\
\cline { 2 - 8 } & Huevo & Instar I & Instar II & Instar III & Instar IV & Prepupa & Pupa & Preimaginal \\
\hline $14,0^{\circ} \mathrm{C}$ & 1,00 & 0,43 & 0,49 & 0,23 & 0,40 & 0,50 & 0,00 & 0,00 \\
$17,6^{\circ} \mathrm{C}$ & 1,00 & 0,85 & 0,91 & 0,83 & 0,94 & 0,97 & 0,90 & 0,53 \\
$25,6^{\circ} \mathrm{C}$ & 0,71 & 0,84 & 0,86 & 0,91 & 0,88 & 0,96 & 0,94 & 0,37 \\
$30,0^{\circ} \mathrm{C}$ & 0,95 & 0,87 & 0,89 & 0,97 & 0,95 & 1,00 & 0,98 & 0,67 \\
$32,0^{\circ} \mathrm{C}$ & 0,59 & 0,70 & 0,54 & 0,93 & 0,43 & 0,35 & 0,53 & 0,02 \\
$34,0^{\circ} \mathrm{C}$ & 0,00 & 0,00 & 0,00 & 0,00 & 0,00 & 0,00 & 0,00 & 0,00 \\
\hline
\end{tabular}


de vida de los estados inmaduros obtenidos son muy cercanas a las reportadas por Miller \& Paustian (1992): huevo, entre 14 y $19 \%$; larva, entre 56 y $60 \%$ y pupa, entre el 22 y el $28 \%$, del total del periodo.

Se considera, por lo tanto, que este depredador se puede adaptar muy bien en pisos térmicos comprendidos entre medios, fríos moderados y fríos en épocas secas, en los que se pueden encontrar las especies de presas evaluadas. En ellos, se incluyen una amplia gama de agroecosistemas, en donde la acción entomófaga de $E$. connexa puede resultar de utilidad en programas de control biológico de plagas.
Modelos de desarrollo dependientes de la temperatura: Para evaluar el ajuste de las observaciones a los modelos, se consideraron los criterios planteados por Roy et al. (2002): Altos valores del coeficiente de determinación $\left(\mathrm{R}^{2}\right)$, baja suma de cuadrados de residuales, facilidad para interpretar los parámetros biológicos e inclusión de los valores observados dentro de los intervalos de confianza al 95\%, y la dificultad planteada por estos autores de tener un modelo que describa el comportamiento del desarrollo dependiente de la temperatura, con características de moderación y un número mínimo de parámetros. Al evaluar diferentes modelos, se encontró que los que mejor se ajustaron,

Tabla 3. Ajuste y valores de los parámetros de los modelos de Logan y Brière para cada uno de los estados de E. connexa.

\begin{tabular}{|c|c|c|c|c|c|c|c|c|c|}
\hline \multirow{2}{*}{ Estado } & \multicolumn{5}{|c|}{ Modelo de Logan } & \multicolumn{4}{|c|}{ Modelo de Brière } \\
\cline { 2 - 9 } & $\begin{array}{c}\mathrm{R}^{2} \\
\text { ajustado }\end{array}$ & $T_{M}$ & $\Delta T$ & $\tau$ & $\rho$ & $\begin{array}{c}\mathrm{R}^{2} \\
\text { ajustado }\end{array}$ & $a$ & $T_{o}$ & $T_{L}$ \\
\hline Huevo & 0,985 & 34 & 0,05 & 0,016 & 0,107 & 0,927 & 0,00003 & 11,3 & 34 \\
Instar I & 0,950 & 34 & 1,05 & 0,044 & 0,076 & 0,928 & 0,00004 & 7,7 & 34 \\
Instar II & 0,947 & 34 & 3,74 & 0,012 & 0,166 & 0,945 & 0,00004 & 11,2 & 34 \\
Instar III & 0,953 & 34 & 2,08 & 0,011 & 0,136 & 0,928 & 0,00004 & 11,9 & 34 \\
Instar IV & 0,965 & 34 & 1,76 & 0,021 & 0,092 & 0,951 & 0,00002 & 9,6 & 34 \\
Pupa & 0,949 & 34 & 1,92 & 0,007 & 0,141 & 0,940 & 0,00003 & 14,5 & 34 \\
Preimaginal & 0,996 & 34 & 2,61 & 0,001 & 0,143 & 0,992 & $4,60 \mathrm{E}-06$ & 13,7 & 34 \\
\hline
\end{tabular}

$\rho$ : la tasa de incremento hasta la temperatura óptima; $T_{M}$ : máxima temperatura, donde el proceso de vida no puede continuar por un periodo prolongado de tiempo; $\Delta T$ : diferencia entre la $T_{M}$ y la temperatura optima; $\tau$ : variable resultante del cociente resultante entre dividir la diferencia entre $T_{M}$ y $T$ sobre $\Delta T ; T_{0}$ : temperatura umbral inferior de desarrollo; a: constante empírica; $T_{L}$ : temperatura letal. Adicionalmente, se realizaron validaciones sobre los residuales de cada estado en cada uno de los dos modelos evaluados para verificar el ajuste.

para todos los estados e instares estudiados, fueron los descritos por la segunda ecuación de Logan et al. (1976) y por la primera ecuación de Brière et al. (1999). Los valores de $\mathrm{R}^{2}$ y parámetros correspondientes, se resumen en la tabla 3.

Huevo: Considerando lo registrado en la tabla 1, el tiempo de desarrollo del huevo, se reduce casi a la mitad, al incrementar la temperatura de 14 a $17,6^{\circ} \mathrm{C}$ $\mathrm{y}$, algo semejante sucede, al incrementarla de 17,6 a $25,6^{\circ} \mathrm{C}$, mientras que la reducción en el desarrollo es casi del $70 \%$ al aumentarla de 25,6 a $30^{\circ} \mathrm{C}$ y, siendo mínima esta reducción, al pasar a los $32^{\circ} \mathrm{C}$. Al realizar pruebas de ajuste de los resultados obtenidos frente a los modelos de Logan et al. (1976) y Brière et al. (1999) (Tabla 3), se encontraron los mejores resultados con el primero de estos dos modelos teóricos $\left(\mathrm{R}^{2}=\right.$ 0,985). De acuerdo a lo estimado por este modelo, el umbral superior de desarrollo se obtiene a los $34^{\circ} \mathrm{C}$ y una temperatura óptima de $33,95^{\circ} \mathrm{C}$, lo que indica, que se pasa muy fácilmente de la óptima a la letal. La limitación del modelo es el no proporcionar el umbral mínimo de temperatura, razón por la cual, se aplicó el modelo de Brière et al. (1999), que aunque presenta un 
ajuste un poco inferior $\left(R^{2}=0,927\right)$ da una buena idea sobre la temperatura mínima requerida para que el huevo complete su desarrollo, la cual fue de $11,3^{\circ} \mathrm{C}$. Usando la metodología de regresión lineal usada por Miller $\mathcal{E}$ Paustian (1992) con los datos del presente trabajo, se obtiene una temperatura de $11,1^{\circ} \mathrm{C}$ como umbral mínimo, frente a un valor de $9,5^{\circ} \mathrm{C}$, encontrada por ellos. En la figura 1a, se puede observar el comportamiento de la tasa de desarrollo frente a los cambios en la temperatura para este estado.

De los parámetros estimados, se puede deducir que para ambos modelos el umbral máximo de temperatura es de $34^{\circ} \mathrm{C}$, dato que no corresponde con lo reportado por Miller \& Paustian (1992), quienes presentan esta temperatura dentro de la fase creciente del modelo lineal de grados-día, propuesto por ellos. Bajo este modelo, en el presente trabajo, se registró un valor de 53,8 grados-día, valor cercano al encontrado porellos, de 54,9 grados día.

Larva: De los datos presentados en la tabla 1, se puede inferir que el estado de larva pasa por cuatro instares y que tiene en promedio una duración entre 14,5 y 64,8 días, dependiendo de la temperatura. Las figuras $1 \mathrm{~b}$, 1c, 1d y 1e representan el ajuste de los datos de tasas de desarrollo de cada instar al modelo de Logan et al. (1976). Tal y como se refleja en la tabla 3, los valores de $\mathrm{R}^{2}$ para los cuatro instares son iguales o mayores a 0,95 , para el modelo de Logan et al. (1976) y son ligeramente superiores a los del modelo de Brière et al. (1999), los cuales, son mayores a 0,93 en todos los casos.

Primer instar. El modelo de Logan permite estimar que el umbral máximo de temperatura es de $34^{\circ} \mathrm{C}$ y que la temperatura óptima es de $33^{\circ} \mathrm{C}$, lo que refleja nuevamente, como en el caso del estado de huevo, que se pasa muy rápido de las condiciones óptimas, para el desarrollo, a condiciones de temperatura, que pueden ser letales para este instar. De otra parte tenemos que el umbral mínimo de temperatura es de $7,7^{\circ} \mathrm{C}$ (modelo de Brière, tabla 3 ), donde es considerablemente más bajo que el del estado precedente $\left(11,3^{\circ} \mathrm{C}\right)$. Segundo instar. De acuerdo a lo observado en la tabla 3 , se tiene que ambos modelos arrojaron temperaturas letales de $34^{\circ} \mathrm{C}$; la temperatura óptima fue de $30,3^{\circ} \mathrm{C}$, mostrando un espacio más amplio entre las dos temperaturas que para los estados precedentes y reflejando, de esta manera, una mayor tolerancia de este instar a incrementos leves de temperatura por encima de la óptima, antes de detener su desarrollo a la temperatura letal, que sigue teniendo el mismo valor que para el huevo y el primer instar. De otra parte, el umbral mínimo de temperatura estimado por el modelo de Brière fue de $11,2^{\circ} \mathrm{C}$, muy cercano al del estado de huevo. Tercer instar. Ambos modelos teóricos mostraron una estimación semejante de la temperatura letal de $34^{\circ} \mathrm{C}$. La temperatura óptima, que refleja la máxima tasa de desarrollo alcanzada fue de $31,9^{\circ} \mathrm{C}$, lo cual, indica que existe un margen de $2,1^{\circ} \mathrm{C}$ entre estos dos valores de temperatura. Un espacio más pequeño que en el instar anterior, pero que de alguna forma presenta una mayor tolerancia en condiciones de cría, en las que se dificulta mantener pequeñas oscilaciones en la temperatura. Por otro lado, en el extremo izquierdo de la curva del modelo de Brière, se encontró un umbral mínimo de temperatura de $11,9^{\circ} \mathrm{C}$, para el tercer instar. Cuarto instar. Haciendo un uso semejante de los dos modelos teóricos al de los estados previos, se tiene una temperatura máxima letal de $34^{\circ} \mathrm{C}$ y una temperatura óptima, mostrando la máxima tasa de desarrollo a $32,2^{\circ} \mathrm{C}$, con una tolerancia de $1,8^{\circ} \mathrm{C}$, entre las dos condiciones de temperatura. Adicionalmente, la estimación de la temperatura mínima para el desarrollo del cuarto instar de $E$. connexa es de $9,6^{\circ} \mathrm{C}$; este dato es cercano al de $9,9^{\circ} \mathrm{C}$ reportado por Miller $\&$ Paustian (1992), para la totalidad del estado de larva, pero inferior al de los otros instares.

En general, el estado de larva muestra un umbral superior de de $34^{\circ} \mathrm{C}$ y un umbral mínimo, que varía entre 7,7 y $11,9^{\circ} \mathrm{C}$, siendo el primer instar el que tolera la temperatura más baja y el tercero el más sensible a las bajas temperaturas. El margen, que proporciona la más alta tasa de desarrollo y en la que se limita ésta por completo, oscila entre 1,1 y $3,7^{\circ} \mathrm{C}$ (Tabla 3), siendo más estrecho para el primer instar y más amplio para el segundo. Esta información resulta de gran utilidad para el establecimiento de las condiciones apropiadas de cría y para la comprensión del comportamiento de la población, a través del tiempo, en condiciones naturales, en las que no se tiene control de las variaciones de la temperatura.

Pupa: La figura $1 \mathrm{f}$, representa el comportamiento observado y ajustado al modelo de Logan de la tasa de desarrollo del estado de pupa, dependiente de la temperatura. Se definió que para este estado ambos modelos arrojaron una estimación de $34^{\circ} \mathrm{C}$, como la temperatura máxima, que no muestra desarrollo, con un 

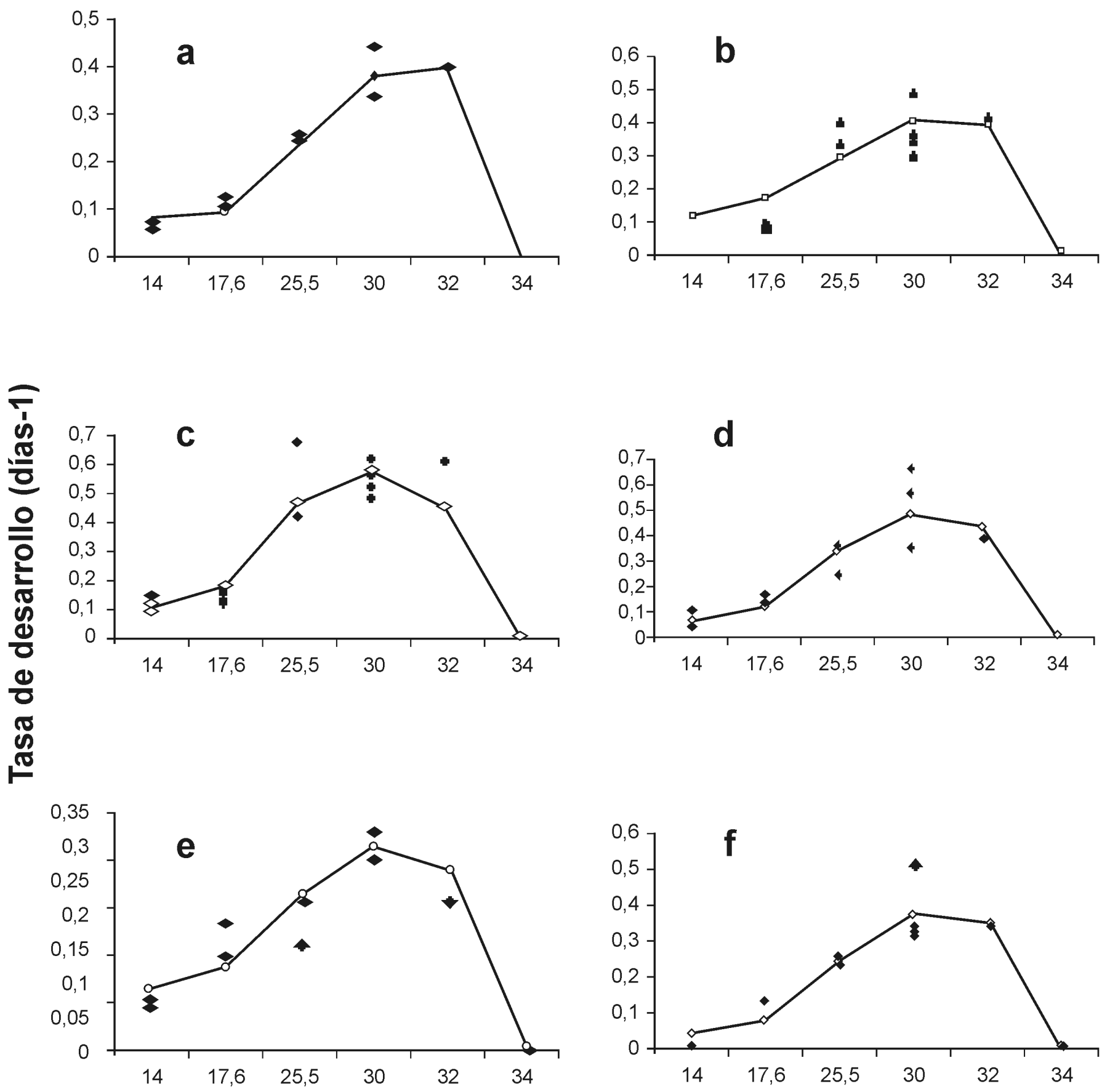

Temperatura $\left({ }^{\circ} \mathrm{C}\right)$

Figura 1. Modelos de tasa de desarrollo dependientes de la temperatura para cada uno de los estados de E. connexa. a, huevo; b, larva de l instar; c, larva de II instar; d, larva de III instar; e, larva de IV instar; f, pupa. Los rombos negros corresponden a los promedios de las diferentes muestras, mientras que los círculos y la línea representan los datos teóricos, ajustados por el modelo de Logan et al. (1976). 
margen de $1,9^{\circ} \mathrm{C}$ entre ésta y la óptima de máxima tasa de desarrollo, que se estimó en $32,1^{\circ} \mathrm{C}$. La temperatura mínima requerida estimada por la ecuación de Brière fue de $14,5^{\circ} \mathrm{C}$. Este último valor sorprende, dado que la pupa se supone como un estado de transformación que, al no requerir alimento, muchas veces tolera condiciones ambientales más extremas, como reportan Miller $\mathcal{E}$ Paustian (1992), quienes encontraron un umbral inferior para la pupa, entre 3,6 y $4^{\circ} \mathrm{C}$. Este dato tampoco es consecuente con la información de Miller (1995), quien afirma que a $4^{\circ} \mathrm{C}$, se pueden almacenar pupas hasta por tres semanas.

Total fase preimaginal: El comportamiento de la tasa de desarrollo desde huevo hasta adulto frente a la temperatura, se ajusta al modelo de Logan y está representado en la figura 2 . El tiempo total desde la oviposición hasta la emergencia del adulto varío entre 18,3 y 50,4 días, para temperaturas entre los 17,6 y $32^{\circ} \mathrm{C}$, de acuerdo con lo presentado en la tabla 2 . El desarrollo preimaginal no se completó a los $14^{\circ} \mathrm{C}$ y ninguno de los estados culminó a $34^{\circ} \mathrm{C}$. De otra parte, se halló un ajuste excelente entre los valores observados y los estimados por el modelo de Logan $\left(R^{2}=0,996\right)$. A partir de éste, se estimó que el umbral máximo de temperatura fue de $34^{\circ} \mathrm{C}$, mientras que la temperatura óptima para la tasa de desarrollo fue de $31,4^{\circ} \mathrm{C}$. El umbral mínimo de temperatura para el total de esta fase fue de $13,7^{\circ} \mathrm{C}$ (de acuerdo con Brière, que mostró un $\mathrm{R}^{2}=0,992$ ). Por esta razón, considerar los valores de los parámetros de umbral mínimo y temperatura óptima para la tasa de desarrollo, de forma global, para la fase preimaginal, no es lo más adecuado, ya que cada estado inmaduro y cada instar larval presentan valores diferentes; el establecimiento de condiciones de cría masiva deben considerar los más altos valores de la temperatura mínima y los más bajos de la temperatura óptima.

Adicionalmente, si con estos datos se realiza un análisis semejante al presentado por Miller E Paustian (1992), se tiene que el umbral mínimo de temperatura es de $13,4^{\circ} \mathrm{C}$ $\left(4,2^{\circ} \mathrm{C}\right.$ mayor que el valor hallado por estos autores) y los grados día son de 259,4 el cual, es muy semejante a 259,1 , reportado por los mismos investigadores.

Una interpretación ecológica de la diferencia de estos valores para cada uno de los estados puede ser la de una capacidad diferente de tolerar temperaturas extremas y el de temperaturas óptimas entre los diferentes estados, con el fin de imprimir heterogeneidad en el desarrollo

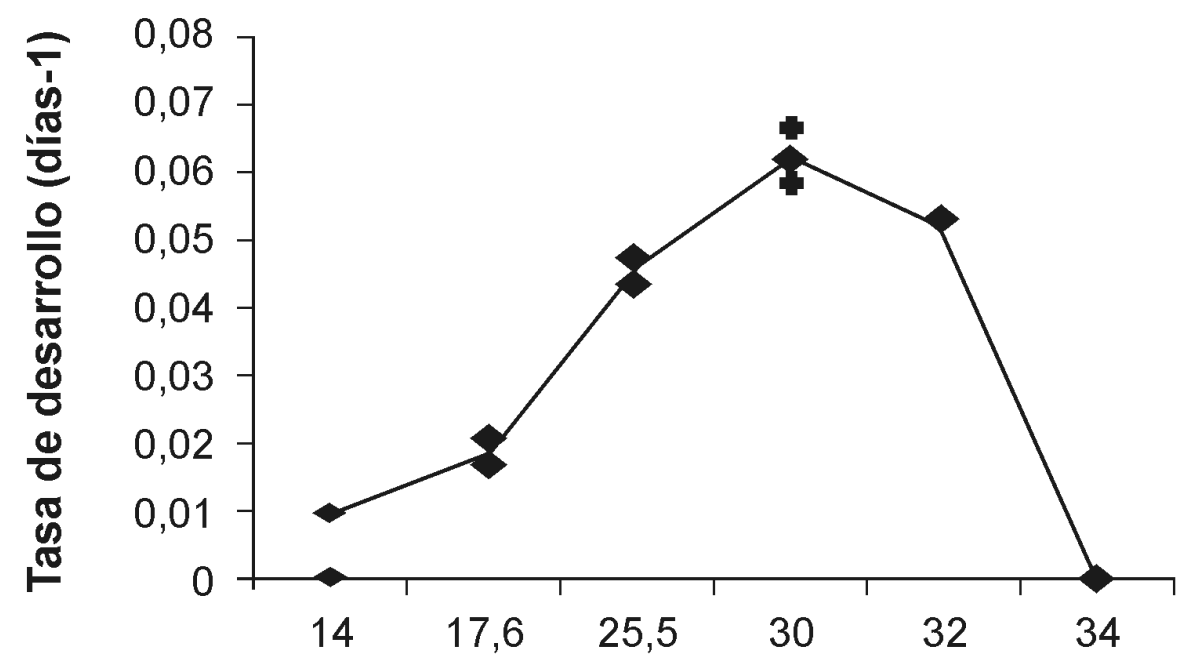

Temperatura $\left({ }^{\circ} \mathrm{C}\right)$

Figura 2. Efecto de la temperatura sobre la tasa de desarrollo desde huevo hasta la emergencia del adulto de E. connexa. Los rombos representan los valores promedio de cada muestra, mientras que los círculos y la línea indican el comportamiento de esta variable ajustado, mediante el modelo de Logan et al. (1976). 
dependiente de la temperatura y favorecer la superposición de estados. Esto garantiza una permanencia continua de la población, así sea a bajas densidades y aún en condiciones ambientales desfavorables. Esta explicación, se puede ver reforzada, además, por la alta variabilidad de las tasas de desarrollo de cada estado, reflejados en altos coeficientes de variación, dentro de cada estado y para cada temperatura.

Las condiciones óptimas de desarrollo para la cría de este insecto deben considerar, simultáneamente, las temperaturas que pueden proporcionar las tasas de desarrollo más altas y la mayor supervivencia, para cada uno de los estados de desarrollo. Para las condiciones en las que se realizó este trabajo, la temperatura óptima de desarrollo estaría entre 25,6 y los $30^{\circ} \mathrm{C}$.

Los resultados se consideran relevantes para el uso de $E$. connexa en programas de control biológico, pues la existencia de un rango óptimo de temperatura entre los 20 y $30^{\circ} \mathrm{C}$ para todos los estados, con tolerancia a cambios ligeros por encima y por debajo de estos valores, es favorable. Ésto, unido al amplio espectro de presas puede facilitar el establecimiento de las poblaciones del coccinélido en ecosistemas y agroecosistemas de clima medio y frío moderado, previos estudios de su capacidad competitiva con otras especies de depredadores existentes y de otras interacciones con otras poblaciones naturales de insectos y ácaros en los lugares en donde se libere. En zonas donde las fluctuaciones de temperatura exceden los umbrales mínimos y/o máximos por lapsos prolongados, como lo son las zonas frías en épocas de verano, donde se presentan temperaturas cercanas a $0^{\circ} \mathrm{C}$ durante las noches, o en épocas de invierno con días lluviosos y permanentemente nublados y temperaturas que no exceden los $14^{\circ} \mathrm{C}$, el uso de este depredador implicaría, necesariamente, la liberación del mismo, en forma masiva, en épocas de altas densidades de especies presa y hasta cuando reaparezcan las condiciones climáticas favorables.

Se concluye que la temperatura como factor climático no solo determina la disponibilidad de alimento para el depredador, sino también afecta la supervivencia de sus poblaciones. La frecuente presencia de $E$. connexa en clima frío representa un potencial para ser usado en control biológico durante épocas cálidas y que, por su capacidad de adaptación, se perfila también como insecto benéfico en cultivos bajo invernadero y en clima medio.
AGRADECIMIENTOS: Los autores hacen manifiesta su gratitud a la U.D.C.A y a sus directivas por el apoyo y la financiación; al Dr. Guillermo Sánchez por su ayuda en la confirmación de las especies de áfidos y al Dr. Miguel Santiago Serrano por su colaboración. Conflicto de intereses: El manuscrito fue preparado y revisado con la participación de todos los autores, quienes declaramos que no existe ningún conflicto de intereses que ponga en riesgo la validez de los resultados presentados.

\section{BIBLIOGRAFÍA}

1. ARIAS DE LAVALLE, G.; COSTAMAGNA, A.C. 1994. Study on the intake capacity of different instars of Eriopis connexa Germ. supplied with Alabama argillacea L (Huebner) eggs. Summaries of the Biological Control Symposium. Granados, Brazil. s.p.

2. ATLIHAN, R.; CHI, H. 2008. Temperature-dependent development and demography of Scymnus subvillosus (Coleoptera: Coccinellidae) reared on Hyalopterus pruni (Homoptera: Aphididae). J. Econ. Entomol. 101(2):325-333.

3. BRIÈRE, J.F.; PRACROS, P.; LE ROUX, A.; PIERRE, J.S. 1999. A novel rate model of temperature dependent development for arthropods. Environ. Entomol. 28 (1):22-29.

4. HERRERA, A.M.; DAHLSTEN, D.D.; TOMIC-CARRUTHERS, N.; CARRUTHERS, N.I. 2005. Estimating temperature-dependent developmental rates of Diorhabda elongata (Coleoptera: Chrysomelidae), a biological control agent of saltcedar (Tamarix spp.). Environ. Entomol. 34(4):775-784.

5. LACTIN, D.J.; HOLLIDAY, N.J.; JOHNSON, D.L.; CRAIGEN, R. 1995. Improved rate model of temperature-dependent development by arthropods. Environ. Entomol. 24(1):68-75.

6. LOGAN, J.A.; WOLLKIND, D.J.; HOYT, S.C.; TANIGOSHI, L.K. 1976. An analytic model for description of temperature dependent rate phenomena in arthropods. Environ. Entomol. (6):1133-1140. 
7. LÓPEZ-ÁVILA, A.; ESPITIA-MALAGÓN, E. 2000. Plagas y benéficos en el cultivo de la papa en Colombia. Boletín Técnico divulgativo. MIP. CorpoicaPRONATTA. Produmedios. Bogotá D.C. 35p.

8. MILLER, 1995. A comparison of techniques for laboratory propagation for South American ladybeatle, Eriopis connexa (Coleoptera: Coccinellidae). Biological control: Theory and Applications in pest management. 5(3):462-465.

9. MILLER, J.C.; PAUSTIAN, J.W. 1992. TemperatureDependent Development of Eriopis connexa (Coleoptera: Coccinellidae). Environ. Entomol. 21(5):1139-1142.

10. MORRIS, R.F.; FULTON, W.C. 1970. Models for the development and survival of Hypantria cunea in relation to temperature and humidity. Mem. Entomol. Soc. Can. 70:1-60.

11. OBRYCKI, J.J.; KRING, T.J. 1998. Predaceous Coccinellidae in biological control. Ann. Rev. Entomol. 43:295-321.

12. OHASHI, D.V.; URDAMPILLETA, J.D. 2003. Interacción entre insectos perjudiciales y benéficos en el cultivo del tabaco de Misiones, Argentina. Rev. Invest. Agr. 32(2):113-124.
13. ROCHA DE MORAES, R.; LEK, A.E.; BELARMINO, E.C. 1991. Inimigos naturais de Rachiplusia nu (Guenée, 1852) e Pseudoplusia includens (Walter, 1857) (Lepidoptera: Noctuidae). Pesq. Agropec. Bras. 26(1):57-64.

14. ROY, M.; BORDEOR, J.; CLOUTIER, C. 2002. Relationship between temperature and developmental rate of Stethorus punctillum (Coleoptera: Coccinellidae) and its prey Tetranychus mcdanieli (Acarina: Tetranychidae). Environ. Entomol. 31(1):177-187.

15. SAS INSTITUTE. 2000. SAS user's guide, versión 8.0. SAS Institute, Cary, NC.

16. VERGARA, R. 1993. El control biológico de insectos- plagas en el cultivo de la papa: de la teoría a la práctica. En: Control Biológico en Colombia, historia, avances y proyectos. $1^{\text {a }}$ Ed. Palacios, F.; Arciniégas, I.; Astudillo, A.M. (Eds.) Palmira, Valle del Cauca. 282p.

17. ZILAHI-BALOGH, G.M.G; SALOM, S.M.; KOK, L.T. 2003. Temperature-dependent Development of the Specialist Predator Laricobius nigrinus (Coleoptera: Derodontidae). Environ. Entomol. 32(6):1322-1328.

Recibido: Agosto 25 de 2009

Aceptado: Noviembre 6 de 2009 